\title{
Research Method of Human Speech System Evolution Based on Complex Social Computing
}

\author{
Jian Yun \\ College of Humanities and Communications, Shanghai Normal University, Shanghai, China \\ College of Computer Science and Technology, Dalian Nationalities University, Dalian, China \\ Email: yunjianm@163.com \\ Chunxia Wang \\ College of Computer Science and Technology, Dalian Nationalities University, Dalian, China
}

\begin{abstract}
In recent years, computing technology has been involved in the field of synchronic phonetics. However, in the research of diachronic phonetics, modern computing technology is always hard to intervene. Human speech system involving a high degree of complexity and many factors, is a typical complex system. The traditional phonetics research methods can not adapt to the characteristics nonlinear complexity of human speech system. Social characteristics in human speech system should be thought as research variables, and non-traditional human speech system evolution research model based on complex system should be applied to diachronic phonetics. First, many basis of this non-traditional research method are given. Then, non-traditional research model for human speech system evolution is proposed. The above work offers some effective intelligent supports for research of diachronic phonetics.
\end{abstract}

Index Terms-complex system, social computing, human speech system evolution, diachronic phonetics, parallel system, multi-agent

\section{INTRODUCTION}

Diachronic linguistics, also known as evolutionary linguistics, mainly researches on the experienced change from ancient to modern. Diachronic linguistics chronologically narrates the history of one language based on literature textual, or rebuilds a language by comparison. The main concern of diachronic linguistics is carding Language family tree and reconstructing the language with contrast. Since the 1950s, there have been some more effective new diachronic linguistics research method or technology, such as, vocabulary proliferation theories(Shiyuan W,1969), tone genetic linguistics(Matisoff J,1973), glottochronology(Swadesh M,1952), remote comparison methods and language variations theories (Labov W,1994,2001).Diachronic linguistics has achieved great success in the Indo-European languages. However, due to the lack of historical literature research on Sino-Tibetan languages, diachronic linguistics research encountered in unprecedented resistance. Therefore, the authors consider that establishing universal suitable diachronic evolution model to replace pure empirical speculation can make the research of language evolution more uniform, objective and scientific. Whether the rapid development of computer science can be involved in the field of research of language evolution? As we all know, language is divided into speech, grammar and semantics. Speech is more measurability. So speech can be digitized is the first reason that we research the language evolution through it. For the other part, 'Speech first in language evolution' is obvious(Carstairs M,1999;Jackendoff R,2002). No matter in the early human, or individuals in early childhood, the change on speech is always the pioneer of language evolution. And we can infer the panorama of language evolution from the speech change. Based on the above, computer modeling on human speech system evolution is the logical starting point on the research of human language evolution, and computer technology make the research of speech evolution more practical operability. We have the reason to believe that computer modeling of the evolution of speech system can go farther on the research of language evolution.

The authors argue that human speech system is a typical complex system, and base on the social calculation method with computer modeling as the technical means, give a research method of speech system evolution.

\section{DiACHRONIC PHONETICS AND SOCIAL COMPUTING}

From the early 19th century, through two hundred years of development, synchronic phonetics has formed a set of quantitative research methods, promoting articulatory phonetics, acoustics phonetics, perceptual phonetics and so on. However, modern computing technologies are always hard to be involved in the research of diachronic linguistics. It may be due to the fact that speech is one of the fleeting acoustic signals, ancient human speech cannot be recorded as acoustic signal or preserved, and the historical documents always have defects. Especially when diachronic linguistics encounters some languages (like most of the East Asian language), which is lack of historical material, it lasts more difficult. In this context, establishing diachronic linguistics which can stand the test of the scientific rules with natural 
science theory and method is far more impossible. Social computing is the interdisciplinary of modern computing technology and social science. The fundamental essence of this concept is using the knowledge of social scientific and computing technology to solve varieties of social science issues. There are indications that, with the growing popularity of computing technology, digital analysis of social science issues has become increasingly urgent. Meanwhile, with the development of artificial intelligence and complex system research, carrying out the study of social computing is ripe. The reason why complex systems research provides the basis for social computing, is based on the following facts: human social behavior in modern world is often a complex system, there is no single optimization objective, there was no complete analytical model accurately.

\section{COMPLEX System AND THE COMPLEXITY OF HUMAN SPEECH SYSTEM}

Complex system research is the fusion product of modern system science and nonlinear science. In general, the so called complex system is basing on the medium scale and existing strong coupling in individuals. Human society is complex system full of interactions. This complex system has the following characteristics: (1) There are many persons with the thinking ability in this complex systems. (2) Some individuals in this complex system is randomness, uncertainty and nonlinear. They affect each other. This system is influenced by the environment and, as verse. (3) This complex system has hierarchical structure, and the interest of each level is usually not uniform often and requires coordination. (4) The composition of such complex system contains experience, wisdom and thinking. (5) This complex system is self-organized and dynamics.

From the view of Complexity Science, language (specific structure of language may be speech) is a complex system with many elements. Language fully embodies the distinctive feature of complex systems: (1) Language is peculiar means of human communication. People use speech signals to express ideas and feelings according to certain rules. (2) In speech system, listener and speaker are interrelated. The perception and imitation are influenced by their pronunciation conditions, Sensing capability and the surrounding environment. Through mutual influence between individuals, the whole speech system emerges some particular macro status. (3) With the continuous development of language, the human speech system presents hierarchical structure. The core of this structure is using the less part to drive the more one, for example, there are more than 400 basic syllables in mandarin Chinese, constituting thousands of words to meet the practical needs of communication. More importantly, the state of social system plays a decisive role in speech evolution. When the social structure has changed, people's social status are also changed. This change will affect the speech system. (4) The External environmental situation and physical limitations on their own pronunciation organs will be analyzed by Intelligent individual. (5) From the perspective of system science, the evolution of speech system is a disordered fluctuation by all complex elements, and then achieves new balance. So self-organizing and dynamics is able to explain the evolution of speech system.

\section{Research Method in SPEech System Evolution BASEd on COMPLEX Social Computing}

\section{A. Several Basic Work}

In order to implement the research of speech system evolution based on complex social computing, the following four fundamental works should do:

(1) Complexity analysis of social characteristics in speech system evolution. (1)Complexity analysis of the social characteristics (such as, the political, economic, cultural, religious beliefs, social psychological changes, etc.) in speech system evolution should be carried out. (2)Treat speech system evolution as a complex system, define the boundary conditions, analyze the direction, speed, and nonlinear relationship in system evolution, and do formal description. (3)people's activities in speech system should be logically analysised and formally descripted.

(2) Construction of computer simulation model of speech system evolution. (1)Environment model in speech system evolution should be constructed. (2)Derive speech system evolution model(the unified model consists of attribute model and behavior model) using evolutionary algorithms. Attribute model describes the various attributes, whereas behavior model describes the interaction between the individuals.3(Realize the visual simulation of the model by computer visual simulation technology.

(3) Study the mechanism of speech evolution based on evolutionary model and complexity theory. (1)Speech system evolution is complex nonlinear system. There are lots of emergence phenomenons which can not be explained with reductionism, So we must pay attention to them, and use visual simulation technology to implement the visualization of emergence phenomenons.(2) The brittleness theory should be applied to the research. We need to identify the brittleness element from evolution model with computer simulation technology.

(4) Construction of parallel system. The so-called "parallel system" refers to the interactive operation model with actual system and artificial complex system(Feiyue W,2006). (1)Repeat the process of the speech system evolution can be helpful to recover the rule of speech system evolution.(2)The author thinks that diachronic phonetics should not only face history, not the future. Parallel system can be established with the combination of practical examples of speech system evolution and visualization evolution model. Through contrastive analyses, the trend of human speech system evolution can be predicted and evaluated. 


\section{B. The Basic Research Model Based on Complex Social Computing}

Speech system is a complex social system which reductionism are difficult to resolve its research problems. With this parallel system method, the various complicated factors can be effectively organized, balanced, controlled and managed. This way, the combination of the modeled and real examples, of diachronic and synchronic analyses, of the past and the future can be made. The untraceables can be traced; the unpredictables can be predicted. This untraditional research method should adopted evolutionary algorithms(such as genetic algorithm, the evolution strategy, evolution of planning, particle swarm algorithm, the ant colony algorithm, etc), multi-agent technology and computer visual simulation technology.Agent is the basic composition of the modeling and simulation. macro-level state of population in speech system is emerged through the interaction between multi-Agents. Figure 1 shows the basic research model based on complex social computing.

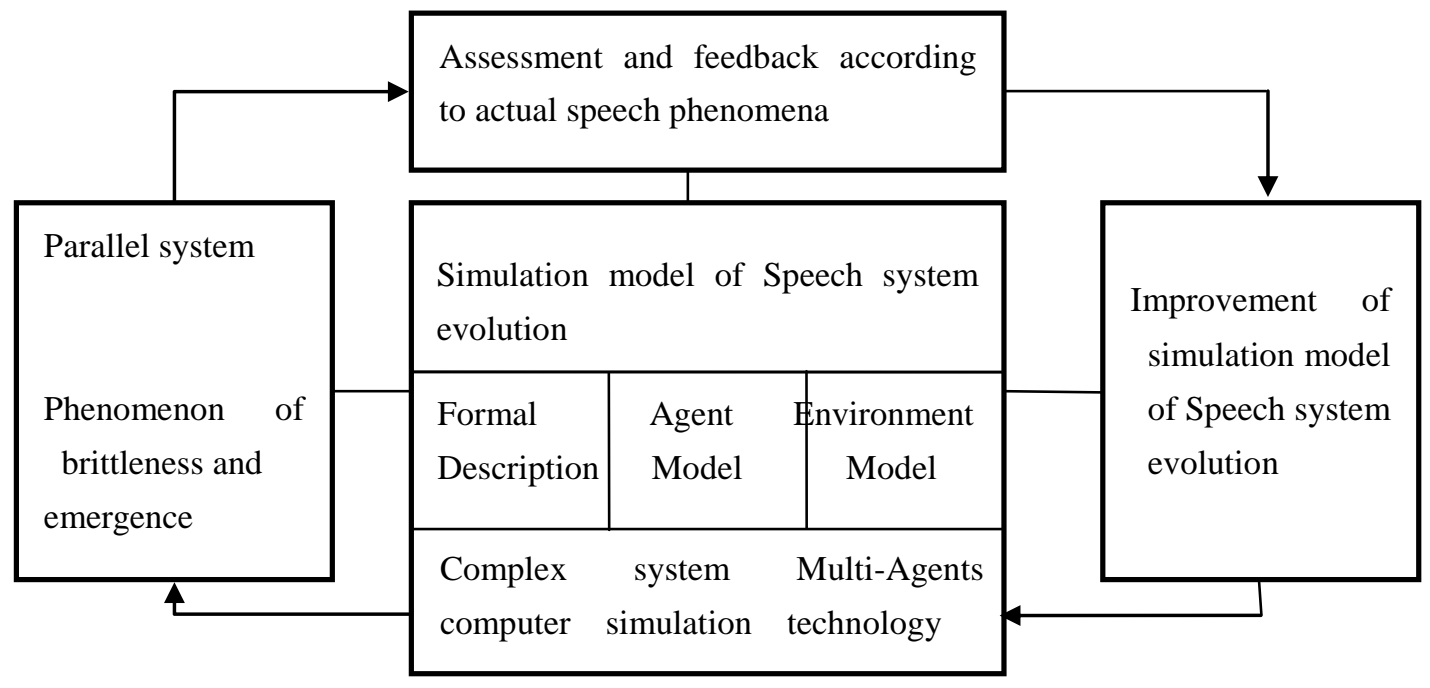

Figure 1. The basic research model based on complex social computing

\section{CONCLUSION}

Human speech is a complex system, so the relationship between micro-level behavior of the individual and macro-state of population level is non-linear, even chaotic in this system. This research model may help us to understand the non-linear relationship between the microscopic behavior and macroscopic state of population level in evolution of speech system. This model should emerge the macro-state similar to state of real speech systems, and illustrates evolution of human speech system.

\section{REFERENCES}

[1] Shiyuan W.(1969). Competing Change as a Cause of Residue. Language. 45.1,19-25

[2] Matisoff J.(1973). Tonogenesis in Southeast Asia, Consonant Types \& Tone. University of Southern California, 71-95.

[3] Swadesh M.(1952).Lexico-statistic dating of prehistoric ethnic contacts: Proceedings of the American Philosophical Societ , $452-463$

[4] Labov W. (1994). Principles of Linguistic Change: Internal Factors. Blackwell Publishing Ltd.

[5] Labov W. (2001). Principles of Linguistic Change: Social Factors. Blackwell Publishing Ltd.

[6] Carstairs M.(1999). The origins of complex language: an inquiry in the evolutionary beginnings of sentences, syllables and truth. Oxford: Oxford University Press.

[7] Jackendoff R.(2002). Foundations of language. Oxford: Oxford University Press.

[8] Feiyue W.(2006). On the Modeling, Analysis, Control and Management of Complex Systems. Complex Systems and Complexity Science. 3.2,26-34.

Jian Yun was born in Baotou, Inner Mongolia, China in 1975. He received his M.A. in Computer Science from Inner Mongol University, China in 2003, and is studying for a doctoral degree in linguistics in the College of Humanities and Communications, Shanghai Normal University, Shanghai, China.

He is currently a lecturer in the College of Computer Science and Technology, Dalian Nationalities University, Dalian, China. His research interests include linguistic evolution and computational linguistics.

Chunxia Wang was born in Ulanqab, Inner Mongolia, China in 1979. She received her M.A. degree in Computer Education from Inner Mongol Normal University, China in 2005.

She is currently a lecturer in the College of Computer Science and Technology, Dalian Nationalities University, Dalian, China. Her research interests include computational linguistics and computer science. 